\title{
Resource Allocation for Secure SWIPT-enabled D2D Communications with $\alpha$ Fairness
}

\author{
Yongjun Xu, Senior Member, IEEE, Bowen Gu, Student Member, IEEE, Dong Li, Senior Member, IEEE, \\ Zhaohui Yang, Member, IEEE, Chongwen Huang, Member, IEEE, Kai-Kit Wong, Fellow, IEEE
}

\begin{abstract}
Device-to-device (D2D) communication is an emerging paradigm that can improve system capacity and spectral efficiency by using cooperative communication coexisting with cellular networks. In spite of these advantages, D2D communication suffers from unfair resource usage, security risks posed by eavesdroppers, and limited energy storage. To deal with these issues, in this paper, we propose a resource allocation algorithm to maximize the security-aware energy efficiency (EE) for D2D users (DUs) in a simultaneous wireless information and power transfer (SWIPT)-enabled D2D communication system with $\alpha$ fairness, where multiple random eavesdroppers are present. In particular, we formulate a multi-objective resource allocation problem by jointly optimizing the transmit power, power-splitting (PS) factors of DUs, and the sub-channel allocation factor under multiple constraints, including the maximum interference power for each cellular user, the maximum transmit power of each DU, the PS factor, and the integer sub-channel assignment. To solve the non-convex problem, an iterative algorithm is developed to obtain the sub-optimal solution. Simulation results verify that the proposed algorithm outperforms benchmark algorithms in terms of balancing secrecy $\mathrm{EE}$ and fairness.
\end{abstract}

Index Terms-Simultaneous wireless information and power transfer, device-to-device communications, resource allocation.

\section{INTRODUCTION}

$\mathbf{I}$ $\mathrm{N}$ the future Internet of Things (IoT) network, the deluge of devices will be widely deployed in various domains to achieve interconnection. However, the limited battery capacity of devices, tremendous energy consumption, and scarce spectrum resources have become stumbling blocks to green

This work was supported by the National Natural Science Foundation of China (61601071, 62071078), Natural Science Foundation of Chongqing (cstc2019jcyj-xfkxX0002), and National Key Research and Development Program (2019YFC1511300). The work of D. Li was supported in part by the Science and Technology Development Fund, Macau SAR, under Grants 0003/2019/A1 and 0110/2020/A3, and in part by the Joint Research Funding Project launched by the Ministry of Science and Technology of the People's Republic of China and the Science and Technology Development Fund, Macau SAR, under Grant 0018/2019/AMJ. (Corresponding authors: Bowen Gu and Dong $\mathrm{Li}$.)

Y. Xu is with the School of Communication and Information Engineering, Chongqing University of Posts and Telecommunications, Chongqing 400065 , China, and also with Chongqing Key Laboratory of Complex Environment Communication, Chongqing 400031, China (e-mail: xuyj@cqupt.edu.cn).

B. Gu and D. Li are with the Faculty of Information Technology, Macau University of Science and Technology, Avenida Wai Long, Taipa, Macau 999078, China (e-mails: gubwww@163.com,dli@must.edu.mo).

Z. Yang is with the Centre for Telecommunications Research, Department of Engineering, King's College London, London WC2B 4BG, U.K. (e-mail: yang.zhaohui@kcl.ac.uk).

C. Huang is with Zhejiang Provincial Key Lab of information processing, communication and networking, Zhejiang University, Hangzhou 310027, China (e-mail: chongwenhuang@zju.edu.cn).

K. K. Wong is with the Department of Electronic and Electrical Engineering, University College London, London WC1E 6BT, U.K. (e-mail: kaikit.wong@ucl.ac.uk).
IoT [1]. Simultaneous wireless information and power transfer (SWIPT) empowers device-to-device (D2D) communication to operate continuously to enable information interaction in case of insufficient battery supply of devices, which gives us the leeway to break the deadlock [2]. On the one hand, in SWIPT, both energy and information signals are carried out from the same radio-frequency (RF) signals, which means that the lifetime of devices can be productively extended with meeting the quality of service (QoS). On the other hand, for D2D communication, information can be shared between user devices without passing through base stations (BSs), where D2D links can reuse the spectrum of cellular users (CUs) to improve spectrum efficiency [3]. Due to its salient merits in terms of the ease of deployment, high spectrum and energy efficiency (EE), SWIPT-enabled D2D communication has been widely concerned.

However, the complicated interference environment, dynamic transmit power, and fluctuating energy harvesting (EH) factors hinder the further implementation of SWIPT-enabled D2D communication networks. Consequently, it is indispensable to design suitable resource allocation strategies. To be specific, in [2], the optimal power control was studied under a single-CU scenario. By extending it to multi-user scenarios, the total ergodic capacity of all D2D users (DUs) was maximized in [3]. However, the energy consumption of the system was ignored in [2] and [3]. To make a balance between transmission rate and energy consumption, the EEbased optimization problems were considered in [4]-[6]. The system EE under the power splitting (PS) mode and the time switching (TS) mode was maximized in [4] and [5], respectively. Besides, in [6], the system EE was maximized by selecting a mode between TS and PS. However, the mentioned works [2]-[6] have not studied secure communication for D2D networks, probably leading to undesirable security risks. To solve this issue, the works [7]-[9] have studied D2D networks in the presence of eavesdroppers (EVEs). The overall sum utility of the system was maximized in [7] under a singleEVE scenario. By extending it to multi-EVE scenarios, the authors in [8] maximized the secrecy throughput of DUs. Considering both resource block (RB) sharing and power control, the total throughput was maximized in [9]. However, the mentioned works [2]-[9] did not take fairness into account. To deal with it, fairness-aware resource allocation problems were studied in [10]-[13]. Based on the max-min fairness, the achievable throughput and secrecy rate of DUs were maximized in [10] and [11], respectively. However, absolute fairness may significantly degrade system performance, which is not desired. To handle this issue, the energy- and spectralefficiency tradeoff with $\alpha$ fairness was investigated in [12] and 


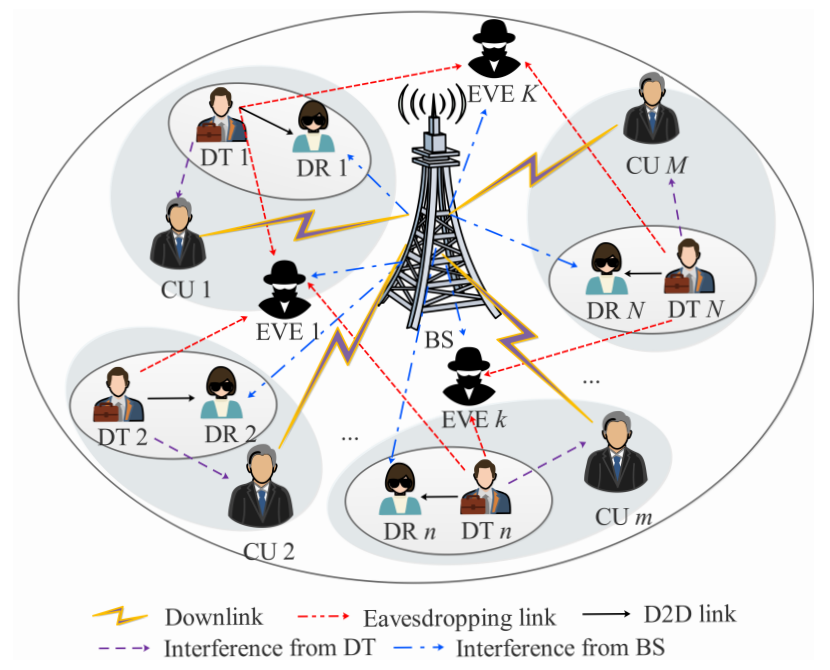

Fig. 1. A SWIPT-enabled D2D network with multiple EVEs.

[13]. However, SWIPT and security issues were not considered in [12] and [13].

Motivated by the above observations, in this paper, we investigate a resource allocation problem for a secure SWIPTenabled D2D communication network with $\alpha$ fairness, where the secrecy EE (SEE) of the system is evaluated. Note that our work differs from the existing works regarding D2D networks in the following aspects: 1) Our work introduces SWIPT into D2D networks from the perspective of EE optimization, which is different from the works in [2], [3], and [7]-[11] that have not focused on EE and the works in [12] and [13] that have not considered SWIPT. 2) The security-aware D2D communication is studied in this work, which is different from the works in [2]-[6], [12], and [13] without taking EVEs into account. 3) The previous works in [2]-[9] have not considered fair resource usage, which is involved in our work. To the authors' best knowledge, little attention has been paid to improving SEE for SWIPT-enabled D2D communications with $\alpha$ fairness. This also implicates that the algorithms proposed by the existing works cannot be directly applied in our work. The contributions of this paper are summarized as follows.

- Our objective is to maximize the system SEE with $\alpha$ fairness by jointly considering the interference threshold of CUs, the maximum transmit power of D2D transmitters (DTs), and user association. The resulting problem is a mixed-integer multi-objective non-convex optimization problem, which is challenging to solve.

- To tackle this non-convex problem, we first convert the original problem into a single-objective problem by using the weighted sum method. Then, an alternating iterative algorithm is developed to deal with the tightly coupled variables due to the PS factors, transmit power, and user association factors. Finally, we derive the closed-form solutions for the corresponding variables.

- Simulation results demonstrate that the proposed algorithm outperforms benchmark algorithms in terms of SEE and fairness.

\section{System Model and Problem Formulation}

\section{A. System Model}

We consider a downlink SWIPT-enabled D2D communication underlaying cellular network with $M$ CUs, $N$ D2D pairs,
TABLE I

\section{SYSTEM PARAMETERS}

\begin{tabular}{c|l}
\hline Notation & Meaning \\
\hline$\beta$ & EH efficiency \\
$\sigma_{0}^{2}$ & EH noise power \\
$\sigma^{2}$ & Background noise power \\
$p_{n}^{\max }$ & Maximum power threshold of DT $n$ \\
$C_{n}^{\mathrm{D}}$ & Circuit power consumption of DR $n$ \\
$I_{m}^{\max }$ & Interference power threshold of CU $m$. \\
$g_{n, m}^{\mathrm{D}}$ & Channel gain between DT $n$ and CU $m$ \\
$\rho_{n, m}^{\mathrm{D}}$ & PS factor of DU $n$ on the sub-channel $m$ \\
$p_{m}^{\mathrm{C}}$ & Transmit power allocated by BS to CU $m$ \\
$h_{n, m}^{\mathrm{D}}$ & Channel gain of DT $n$ on the sub-channel $m$ \\
$p_{n, m}^{\mathrm{D}}$ & Transmit power of DT $n$ on the sub-channel $m$ \\
$x_{n, m}$ & User association factor between DU $n$ and CU $m$ \\
$g_{m}^{\mathrm{C}}$ & Channel gain from BS to DR $n$ on the sub-channel $m$ \\
$g_{m, k}^{\mathrm{E}}$ & Channel gain from BS to EVE $n$ on the sub-channel $m$ \\
$h_{n, m, k}^{\mathrm{E}}$ & Channel gain from DT $n$ to EVE $n$ on the sub-channel $m$ \\
\hline
\end{tabular}

$K$ EVEs, and a BS, as shown in Fig. 1. Each CU occupies one orthogonal sub-channel. In order to improve spectrum efficiency, the sub-channel of the $\mathrm{CU}$ can be reused by a pair of DUs. Each D2D receiver (DR) is equipped with an EH circuit. The received signal at the DR can be divided into the EH signal and the information signal via PS. Denote the sets of CUs, DUs, and EVEs by $\mathcal{M}=\{1,2, \ldots, M\}(\forall m \in \mathcal{M})$, $\mathcal{N}=\{1,2, \cdots, N\}(\forall n \in \mathcal{N})$, and $\mathcal{K}=\{1,2, \cdots, K\}$ $(\forall k \in \mathcal{K})$, respectively. The notations used in this paper are shown in Table I.

The achievable rate and the harvested energy of DR $n$ are, respectively, given by

$$
\begin{array}{r}
R_{n}^{\mathrm{D}}=\sum_{m=1}^{M} x_{n, m} \log _{2}\left(1+\frac{\rho_{n, m}^{\mathrm{D}} p_{n, m}^{\mathrm{D}} h_{n, m}^{\mathrm{D}}}{\rho_{n, m}^{\mathrm{D}}\left(\rho_{m}^{\mathrm{C}} g_{m, n}^{\mathrm{d}}+\sigma_{0}^{2}\right)+\sigma^{2}}\right), \forall n, m, \\
E_{n}^{\mathrm{D}}=\beta \sum_{m=1}^{M} x_{n, m}\left(1-\rho_{n, m}^{\mathrm{D}}\right)\left(p_{n, m}^{\mathrm{D}} h_{n, m}^{\mathrm{D}}+\sum_{s=1}^{M} p_{s}^{\mathrm{C}} g_{s, n}^{\mathrm{C}}\right), \forall n, m, s
\end{array}
$$

where $x_{n, m}$ is the user association factor. In particular, $x_{n, m}=$ 1 means that the $n$-th D2D pair occupies the sub-channel of the $m$-th $\mathrm{CU}$; otherwise $x_{n, m}=0$. Assuming that the distance between different DUs is relatively far apart so that the received power from other DUs can be neglected [4]. Thus, the harvested energy of the $n$-th DR is from the $n$-th DT and the BS, as shown in (2).

The achievable rate of EVE $k$ intercepting the information of DT $n$ can be expressed as

$$
R_{n, k}^{\mathrm{E}}=\sum_{m=1}^{M} x_{n, m} \log _{2}\left(1+\frac{p_{n, m}^{\mathrm{D}} h_{n, m, k}^{\mathrm{E}}}{p_{m}^{\mathrm{C}} g_{m, k}^{\mathrm{E}}+\sigma_{0}^{2}+\sigma^{2}}\right), \forall n, m, k
$$

The EVEs are assumed to be non-collusive [14], so that the secrecy rate of DU $n$ can be written as

$$
R_{n}^{\mathrm{S}}=\left[R_{n}^{\mathrm{D}}-\max _{k \in \mathcal{K}} R_{n, k}^{\mathrm{E}}\right]^{+}, \forall n, k,
$$

where $[x]^{+}$denotes $\max (0, x)$.

Correspondingly, the power consumption of DU $n$ is given by

$$
E_{n}^{\mathrm{C}}=\left[\sum_{m=1}^{M} x_{n, m} p_{n, m}^{\mathrm{D}}+C_{n}^{\mathrm{D}}-E_{n}^{\mathrm{D}}\right]^{+}, \forall n, m .
$$




\section{B. Problem Formulation}

We adopt the $\alpha$-fair utility to model fairness [13], which is given by

$$
u_{\alpha}\left(R_{n}^{\mathrm{S}}\right)=\left\{\begin{array}{l}
\ln \left(R_{n}^{\mathrm{S}}\right), \text { if } \alpha=1, \\
\frac{\left(R_{n}^{\mathrm{S}}\right)^{1-\alpha}}{1-\alpha}, \text { if } \alpha \neq 1, \alpha \geq 0,
\end{array}\right.
$$

where $\alpha$ represents the fairness factor. To be specific, the utility function $U_{\alpha}(\cdot)$ represents zero fairness with $\alpha=0$, proportional fairness with $\alpha=1$, harmonic mean fairness with $\alpha=2$, and absolute fairness with $\alpha=+\infty$ [15].

To investigate the tradeoff between energy consumption and secrecy rate with $\alpha$ fairness, a multi-objective optimization problem can be formulated as

$$
\begin{aligned}
& \quad \max _{p_{n, m}^{\mathrm{D}}, x_{n, m}, \rho_{n, m}^{\mathrm{D}}} \sum_{n=1}^{N} u_{\alpha}\left(R_{n}^{\mathrm{S}}\right) \\
& \quad \max _{p_{n, m}^{\mathrm{D}}, x_{n, m}, \rho_{n, m}^{\mathrm{D}}}-\sum_{n=1}^{N} E_{n}^{\mathrm{C}} \\
& \text { s.t. } \quad C_{1}: \sum_{n=1}^{N} x_{n, m} p_{n, m}^{\mathrm{D}} g_{n, m}^{\mathrm{D}} \leq I_{m}^{\max }, \forall n, m, \\
& \quad C_{2}: \sum_{m=1}^{M} x_{n, m} \leq 1, x_{n, m} \in\{0,1\}, \forall n, m, \\
& C_{3}: \sum_{m=1}^{M} x_{n, m} p_{n, m}^{\mathrm{D}} \leq p_{n}^{\max }, \forall n, m, \\
& \quad C_{4}: 0<\rho_{n, m}^{\mathrm{D}}<1, \forall n, m .
\end{aligned}
$$

Note that $C_{1}$ is used to protect the QoS of $\mathrm{CU} m, C_{2}$ is the user association constraint, $C_{3}$ denotes the maximum transmit power and $C_{4}$ is the range of the PS factor.

\section{Resource Allocation Algorithm}

It can be easily verified that problem (7) is non-convex due to the non-convex constraints and the multi-objective function as well as the binary variables. In what follows, we first transform the original problem into a convex one by using slack variables and variable substitution. Then, the closed-form solution is deduced by using the Lagrange dual method.

\section{A. Problem Transformation}

Since the objective function contains a max-min secrecy rate (e.g., $R_{n}^{\mathrm{S}}$ ), it's hard to solve problem (7) directly. To make it more tractable, we introduce a new slack variable $\tau_{n}$ [16], and problem (7) can be reformulated as

$$
\begin{aligned}
& \max _{p_{n, m}^{\mathrm{D}}, x_{n, m}, \rho_{n, m}^{\mathrm{D}}, \tau_{n}} \sum_{n=1}^{N} u_{\alpha}\left(\tau_{n}\right) \\
& \quad \max _{p_{n, m}^{\mathrm{D}}, x_{n, m}, \rho_{n, m}^{\mathrm{D}}, \tau_{n}}-\sum_{n=1}^{N} E_{n}^{\mathrm{C}} \\
& \text { s.t. } C_{1}-C_{4}, \\
& \quad C_{5}: R_{n}^{\mathrm{D}}-R_{n, k}^{\mathrm{E}} \geq \tau_{n}, \forall n, k .
\end{aligned}
$$

However, problem (8) is still intractable to address since the value ranges of the two optimization goals are quite different. To deal with it, the two objective functions of problem (8) are normalized as follows

$$
\begin{aligned}
& \max _{p_{n, m}^{\mathrm{D}}, x_{n, m}, \rho_{n, m}^{\mathrm{D}}, \tau_{n}} \frac{\sum_{n=1}^{N} u_{\alpha}\left(\tau_{n}\right)-U_{\min }^{\alpha}}{U_{\max }^{\alpha}-U_{\min }^{\alpha}}, \\
& \max _{p_{n, m}^{\mathrm{D}}, x_{n, m}, \rho_{n, m}^{\mathrm{D}}, \tau_{n}}-\frac{\sum_{n=1}^{N} E_{n}^{\mathrm{C}}}{\sum_{n=1}^{N} E_{n}^{\max }},
\end{aligned}
$$

where $U_{\max }^{\alpha}$ and $U_{\min }^{\alpha}$ are the maximum and minimum total secrecy rate, and $E_{n}^{\max }$ is the maximum power consumption, given by

$$
\begin{gathered}
U_{\max }^{\alpha}=\max _{x_{n, m}, \rho_{n, m}^{\mathrm{D}}, \tau_{n}, \rho_{n, m}^{\mathrm{D}},} \sum_{n=1}^{N} u_{\alpha}\left(\tau_{n}\right), \forall n, m, \\
U_{\min }^{\alpha}=\left\{\begin{array}{l}
\sum_{n=1}^{N} \ln (\theta), \text { if } \alpha=1, \\
\sum_{n=1}^{N} \frac{\theta^{1-\alpha}}{1-\alpha}, \text { if } \alpha \neq 1, \alpha \geq 0,
\end{array}\right. \\
E_{n}^{\max }=\max _{x_{n, m}, \rho_{n, m}^{\mathrm{D}}, \tau_{n}, \rho_{n, m}^{\mathrm{D}}} p_{n, m}^{\mathrm{D}}+C_{n}^{\mathrm{D}}-E_{n}^{\mathrm{D}}, \forall n, m,
\end{gathered}
$$

where $U_{\max }^{\alpha}$ can be obtained by the network utility maximization algorithm in [12]. For $U_{\min }^{\alpha}$, the secrecy rates of all DUs are bigger than $\theta$, where $\theta$ stands for a sufficiently small value, satisfying $\theta \in\left(0, \tau_{n}\right]$ [13].

Then, the weighted sum method [17] is used to transform problem (8) with multi-objective functions into one with a single-objective function. Thus, we can get the equivalent optimization problem, i.e.,

$$
\begin{aligned}
& \max _{p_{n, m}^{\mathrm{D}}, x_{n, m}, \rho_{n, m}^{\mathrm{D}}, \tau_{n}} \mathcal{F}=\frac{w\left(\sum_{n=1}^{N} u_{\alpha}\left(\tau_{n}\right)-U_{\min }^{\alpha}\right)}{U_{\max }^{\alpha}-U_{\min }^{\alpha}}-\frac{(1-w) \sum_{n=1}^{N} E_{n}^{\mathrm{C}}}{\sum_{n=1}^{N} E_{n}^{\max }} \\
& \text { s.t. } C_{1}-C_{5},
\end{aligned}
$$

where $w \in[0,1]$ is the weight factor. To be specific, given a fixed $\alpha$, we can investigate the tradeoff between the secrecy rate and the energy consumption by adjusting $w$.

\section{B. Algorithm Design}

However, problem (13) is still non-convex due to the tightly coupled variables. To make it tractable, we obtain the variable $\rho_{n, m}^{\mathrm{D}}$ through a monotonic optimization approach at first. Then, problem (13) can be solved in an alternating iterative manner. Specifically, assuming that the $n$-th DU reuses the sub-channel of the $m$-th $\mathrm{CU}$, according to $C_{5}$, we have

$$
\rho_{n, m}^{\mathrm{D}} \geq \frac{\sigma^{2}\left(2^{\mu_{n, m}}-1\right)}{p_{n, m}^{\mathrm{D}} h_{n, m}^{\mathrm{D}}-\left(2^{\mu_{n, m}}-1\right)\left(p_{m}^{\mathrm{C}} g_{m, n}^{\mathrm{C}}+\sigma_{0}^{2}\right)}, \forall n, m,
$$

where $\mu_{n, m}=\tau_{n}+\log _{2}\left(1+\frac{p_{n, m}^{D} h_{m, k}^{E}}{p_{m}^{\mathrm{C}} g_{m, k}^{\mathrm{E}}+\sigma_{0}^{2}+\sigma^{2}}\right)$.

Besides, it can be verified that $\mathcal{F}$ in (13) is a monotonically decreasing function with $\rho_{n, m}^{\mathrm{D}}$. Thus, the optimal PS factor $\rho_{n, m}^{\mathrm{D}, *}$ can be found as

$$
\rho_{n, m}^{\mathrm{D}, *}=\frac{\sigma^{2}\left(2^{\mu},, m-1\right)}{p_{n, m}^{\mathrm{D}} h_{n, m}^{\mathrm{D}}-\left(2^{\mu, m}-1\right)\left(p_{m}^{\mathrm{C}} g_{m, n}^{\mathrm{C}}+\sigma_{0}^{2}\right)} .
$$

From (15), it can be seen that, $\rho_{n, m}^{\mathrm{D}, *}$ can be obtained, in case $p_{n, m}^{\mathrm{D}, *}, x_{n, m}^{*}$ and $\tau_{n}^{*}$ are determined. Proactively, to deal with the couple variables $\left(p_{n, m}^{\mathrm{D}}\right.$ and $\left.x_{n, m}\right)$, we define $y_{n, m}=p_{n, m}^{\mathrm{D}} x_{n, m}$. Based on an alternating optimization 
method, problem (13) with the fixed $\rho_{n, m}^{\mathrm{D}}$ can be rewritten as

$$
\begin{array}{ll}
\max _{y_{n, m}, x_{n}, m}, \tau_{n} & \frac{w\left(\sum_{n=1}^{N} u_{\alpha}\left(\tau_{n}\right)-U_{\min }^{\alpha}\right)}{U_{\max }^{\alpha}-U_{\min }^{\alpha}}-\frac{(1-w) \sum_{n=1}^{N} y_{n}}{\sum_{n=1}^{N} E_{n}^{\max }} \\
\text { s.t. } & \bar{C}_{1}: \sum_{n=1}^{N} y_{n, m} g_{n, m}^{\mathrm{D}} \leq I_{m}^{\max }, \forall n, m, \\
& \bar{C}_{2}: \sum_{m=1}^{M} x_{n, m} \leq 1,0 \leq x_{n, m} \leq 1, \forall n, m, \\
& \bar{C}_{3}: \sum_{m=1}^{M} y_{n, m} \leq p_{n}^{\max }, \forall n, m, \\
& \bar{C}_{5}: \tilde{R}_{n}^{\mathrm{D}}-\tilde{R}_{n, k}^{\mathrm{E}} \geq \tau_{n}, \forall n, k,
\end{array}
$$

where $\quad y_{n} \quad=\quad \sum_{m=1}^{M} y_{n, m}+C_{n}^{\mathrm{D}}+\beta l_{n}, \quad l_{n}=$ $\sum_{m=1}^{M}\left(\rho_{n, m}^{\mathrm{D}}-1\right)\left(y_{n, m}^{\mathrm{D}}+\sum_{s=1}^{M} x_{n, m} p_{s}^{\mathrm{C}} g_{s, n}^{\mathrm{C}}\right), \quad \tilde{R}_{n}^{\mathrm{D}}=$ $\sum_{m=1}^{M} x_{n, m} \log _{2}\left(1+\frac{\rho_{n, m}^{\mathrm{D}} y_{n, m} h_{n, m}^{\mathrm{D}}}{x_{n, m}\left(\rho_{n, m}^{\mathrm{D}}\left(p_{m}^{\mathrm{C}} g_{m, n}^{\mathrm{C}}+\sigma_{0}^{2}\right)+\sigma^{2}\right)}\right)$, and $\tilde{R}_{n, k}^{\mathrm{E}}=$ $\sum_{m=1}^{M} x_{n, m} \log _{2}\left(1+\frac{y_{n, m} h_{n, k}^{\mathrm{E}}}{x_{n, m}\left(p_{m}^{\mathrm{C}} g_{m, k}^{\mathrm{E}}+\sigma_{0}^{2}+\sigma^{2}\right)}\right)$. For $t_{1}>t_{2} \geq 0$, the function $f(x, y) \triangleq x \log _{2}\left(1+\frac{t_{1} y}{x}\right)-x \log _{2}\left(1+\frac{t_{2} y}{x}\right)$ is concave [16]. Therefore, $\bar{C}_{5}$ is a convex constraint, and problem (16) is a convex optimization problem, which can be efficiently solved. Here, to obtain more meaningful insights, the Lagrange duality method is applied to achieve the closed-form solution to problem (16). To be more specific, the Lagrange function of problem (16) with $\bar{C}_{5}$ is

$$
\begin{aligned}
\mathcal{L}\left(x_{n, m}, \tau_{n}, y_{n, m}, \lambda_{n}\right)= & \frac{w\left(\sum_{n=1}^{N} u_{\alpha}\left(\tau_{n}\right)-U_{\min }^{\alpha}\right)}{U_{\max }^{\alpha}-U_{\min }^{\alpha}}-\frac{(1-w) \sum_{n=1}^{N} y_{n}}{\sum_{n=1}^{N} E_{n}^{\max }} \\
& +\sum_{n=1}^{N} \lambda_{n}\left(\tilde{R}_{n}^{\mathrm{D}}-\tilde{R}_{n, k}^{\mathrm{E}}-\tau_{n}\right),
\end{aligned}
$$

where $\lambda_{n}$ is a non-negative Lagrange multiplier. Accordingly, the dual function can be written as

$$
g\left(\lambda_{n}\right)=\left\{\begin{array}{l}
\max _{x_{n, m}, \tau_{n}, y_{n, m}} \mathcal{L}\left(x_{n, m}, \tau_{n}, y_{n, m}, \lambda_{n}\right) \\
\text { s.t. } \bar{C}_{1}-\bar{C}_{3} .
\end{array}\right.
$$

The corresponding dual problem can be formulated as

$$
\begin{aligned}
& \min _{\lambda_{n}} g\left(\lambda_{n}\right) \\
& \text { s.t. } \bar{C}_{1}-\bar{C}_{3} .
\end{aligned}
$$

It can be seen that, in (17) and (18), the dual function consists of two sets of variables: application-layer variable $\tau_{n}$, and physical-layer variables $x_{n, m}, \tau_{n}$, and $y_{n, m}$. Hence, the dual problem is separated into two sub-problems: the applicationlayer optimization problem $g_{1}\left(\lambda_{n}\right)$ and the physical-layer optimization problem $g_{2}\left(\lambda_{n}\right)$, i.e.,

$$
g_{1}\left(\lambda_{n}\right)=\max _{\tau_{n} \geq 0} f\left(\tau_{n}\right)=\frac{w\left(\sum_{n=1}^{N} u_{\alpha}\left(\tau_{n}\right)-U_{\min }^{\alpha}\right)}{U_{\max }^{\alpha}-U_{\min }^{\alpha}}-\sum_{n=1}^{N} \lambda_{n} \tau_{n},
$$

and

$$
g_{2}\left(\lambda_{n}\right)=\left\{\begin{array}{l}
\max _{y_{n, m, x_{n}, m}} \sum_{n=1}^{N} \lambda_{n}\left(\tilde{R}_{n}^{\mathrm{D}}-\tilde{R}_{n, k}^{\mathrm{E}}\right)-\frac{(1-w) \sum_{n=1}^{N} y_{n}}{\sum_{n=1}^{N} E_{n}^{\max }}, \\
\text { s.t. } \bar{C}_{1}-\bar{C}_{3} .
\end{array}\right.
$$

At first, we solve $g_{1}\left(\lambda_{n}\right)$. For the case of $\alpha=0$ and $\alpha>$ 0 , the expressions for $g_{1}\left(\lambda_{n}\right)$ are obtained according to the definition of $u_{\alpha}\left(\tau_{n}\right)$. It can be seen that both $f\left(\tau_{n}\right)$ and $u_{\alpha}\left(\tau_{n}\right)$ are concave functions w.r.t. $\tau_{n}$ when $\alpha>0$ holds. Letting $\left.\frac{\partial f\left(\tau_{n}\right)}{\partial \tau_{n}}\right|_{\alpha>0}=0$, we have

$$
\tau_{n}^{*}=\sqrt[\alpha]{\frac{w}{\lambda_{n}\left(U_{\max }^{\alpha}-U_{\min }^{\alpha}\right)}}, \alpha>0 .
$$

On the other hand, when $\alpha=0$ holds, we have

$$
\tau_{n}^{i+1}=\left[\tau_{n}^{i}-\Delta_{\tau} \times\left.\frac{\partial f\left(\tau_{n}\right)}{\partial \tau_{n}}\right|_{\alpha=0}\right]^{+}, \alpha=0,
$$

where $\left.\frac{\partial f\left(\tau_{n}\right)}{\partial \tau_{n}}\right|_{\alpha=0}=w-\lambda_{n}, i$ denotes the iterative number of $\mathcal{L}(\cdot)$, and $\Delta_{\tau}$ is the step size.

Then, we solve $g_{2}\left(\lambda_{n}\right)$. The Lagrange function of $g_{2}\left(\lambda_{n}\right)$ can be formulated as

$$
\begin{aligned}
\overline{\mathcal{L}}\left(U_{n, m}\right)= & \sum_{n=1}^{N} \lambda_{n}\left(\tilde{R}_{n}^{\mathrm{D}}-\tilde{R}_{n, k}^{\mathrm{E}}\right)+\sum_{n=1}^{N} \psi_{n, m}\left(p_{n}^{\max }-\sum_{m=1}^{M} y_{n, m}\right) \\
& -\frac{(1-w) \sum_{n=1}^{N} y_{n}}{\sum_{n=1}^{N} E_{n}^{\max }}+\sum_{m=1}^{M} \xi_{n, m}\left(I_{m}^{\max }-\sum_{n=1}^{N} y_{n, m} g_{n, m}^{\mathrm{D}}\right) \\
& +\sum_{m=1}^{M} \kappa_{n, m}\left(1-\sum_{n=1}^{N} x_{n, m}\right),
\end{aligned}
$$

where $U_{n, m}=\left\{x_{n, m}, y_{n, m}, \xi_{n, m}, \psi_{n, m}, \kappa_{n, m}\right\} . \xi_{n, m}, \psi_{n, m}$, and $\kappa_{n, m}$ are non-negative Lagrange multipliers. According to the Karush-Kuhn-Tucker conditions [18], we have

$$
p_{n, m}^{\mathrm{D}, *}=\left[\frac{\sqrt{\bar{D}_{n, m}^{2}-4 K_{n, m} D_{n, m} \rho_{n, m}^{\mathrm{D}} h_{n, k}^{\mathrm{E}} h_{n, m}^{\mathrm{D}}}-\bar{D}_{n, m}}{2 D_{n, m} \rho_{n, m}^{\mathrm{D}} h_{n, k}^{\mathrm{E}} h_{n, m}^{\mathrm{D}}}\right]^{+},
$$

where $\bar{D}_{n, m}=D_{n, m} h_{n, k}^{\mathrm{E}} E_{n, m}+D_{n, m} \rho_{n, m}^{\mathrm{D}} h_{n, m}^{\mathrm{D}} J_{n, m}, D_{n, m}=$ $\frac{\ln 2(1-w)}{\lambda_{n} E_{n}^{\max }}\left(1+\beta \rho_{n, m}^{\mathrm{D}} h_{n, m}^{\mathrm{D}}-\beta h_{n, m}^{\mathrm{D}}\right)+\frac{\ln 2}{\lambda_{n}}\left(\xi_{m} g_{n, m}^{\mathrm{D}}+\psi_{n}\right), J_{n, m}=$ $p_{m}^{\mathrm{C}} g_{m, k}^{\mathrm{E}}+\sigma_{0}^{2}+\sigma^{2}, E_{n, m}=\rho_{n, m}^{\mathrm{D}}\left(p_{m}^{\mathrm{C}} g_{m, n}^{\mathrm{C}}+\sigma_{0}^{2}\right)+\sigma^{2}$, and $K_{n, m}=$ $D_{n, m} E_{n, m} J_{n, m}+h_{n, k}^{\mathrm{E}} E_{n, m}-\rho_{n, m}^{\mathrm{D}} h_{n, m}^{\mathrm{D}} J_{n, m}$.

To obtain the optimal $x_{n, m}$, we have

$$
\frac{\partial \overline{\mathcal{L}}\left(U_{n, m}\right)}{\partial x_{n, m}}=\phi_{n, m}-\kappa_{m}=\left\{\begin{array}{l}
<0, x_{n, m}=0, \\
=0,0<x_{n, m}<1, \\
>0, x_{n, m}=1,
\end{array}\right.
$$

where

$$
\begin{aligned}
\phi_{n, m} & =\lambda_{n}\left[\log _{2}\left(1+\frac{\rho_{n, m}^{\mathrm{D}} p_{n, m}^{\mathrm{D}} h_{n, m}^{\mathrm{D}}}{E_{n, m}}\right)-\log _{2}\left(1+\frac{p_{n, m}^{\mathrm{D}} h_{n, k}^{\mathrm{E}}}{J_{n, m}}\right)\right] \\
& -\frac{(1-w)}{E_{n}^{\max }} \beta\left\{\left(\rho_{n, m}^{\mathrm{D}}-1\right)\left(p_{n, m}^{\mathrm{D}} h_{n, m}^{\mathrm{D}}+\sum_{s=1}^{M} p_{s}^{\mathrm{C}} g_{s, n}^{\mathrm{C}}\right)\right\} \\
& -\frac{(1-w)}{E_{n}^{\max }} p_{n, m}^{\mathrm{D}}-\psi_{n} p_{n, m}^{\mathrm{D}}-\xi_{m} p_{n, m}^{\mathrm{D}} g_{n, m}^{\mathrm{D}} .
\end{aligned}
$$

Therefore, the $n$-th DU reuses the sub-channel of the $m$-th $\mathrm{CU}$ according to the largest $\phi_{n, m}$, i.e.,

$$
x_{n, m}=1 \mid m^{*}=\max _{n} \phi_{n, m} .
$$

Based on the subgradient method [18], Lagrange multipliers can be updated by

$$
\lambda_{n}^{i+1}=\left[\lambda_{n}^{i}-\Delta_{\lambda} \times\left(\tilde{R}_{n}^{\mathrm{D}}-\tilde{R}_{n, k}^{\mathrm{E}}-\tau_{n}\right)\right]^{+},
$$




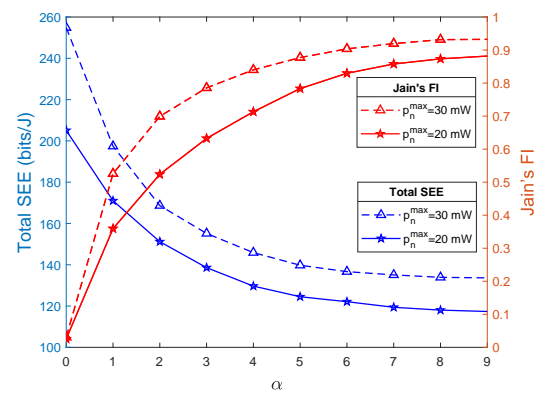

Fig. 2. SEE and FI vs $\alpha$.

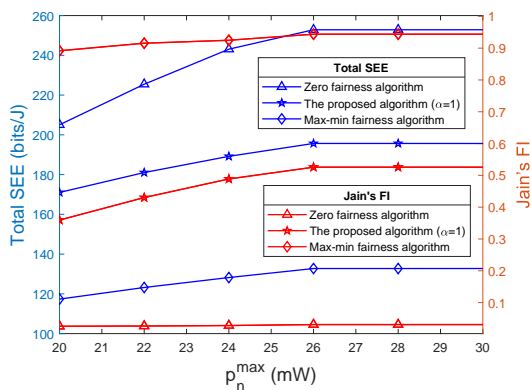

Fig. 3. SEE and FI vs $p_{n}^{\max }$.

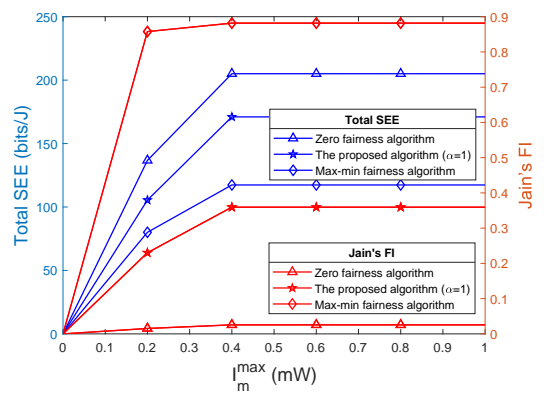

Fig. 4. SEE and FI vs $I_{n}^{\max }$.

$$
\begin{gathered}
\kappa_{n, m}^{t+1}=\left[\kappa_{n, m}^{t}-\Delta_{\kappa} \times\left(1-\sum_{n=1}^{N} x_{n, m}\right)\right]^{+}, \\
\psi_{n, m}^{t+1}=\left[\psi_{n, m}^{t}-\Delta_{\psi} \times\left(p_{n}^{\max }-\sum_{m=1}^{M} y_{n, m}\right)\right]^{+}, \\
\xi_{n, m}^{t+1}=\left[\xi_{n, m}^{t}-\Delta_{\xi} \times\left(I_{m}^{\max }-\sum_{n=1}^{N} y_{n, m} g_{n, m}^{\mathrm{D}}\right)\right]^{+},
\end{gathered}
$$

where $t$ is the iterative number of $\overline{\mathcal{L}}(\cdot), \Delta_{\lambda}, \Delta_{\kappa}, \Delta_{\psi}$, and $\Delta_{\xi}$ are the step sizes.

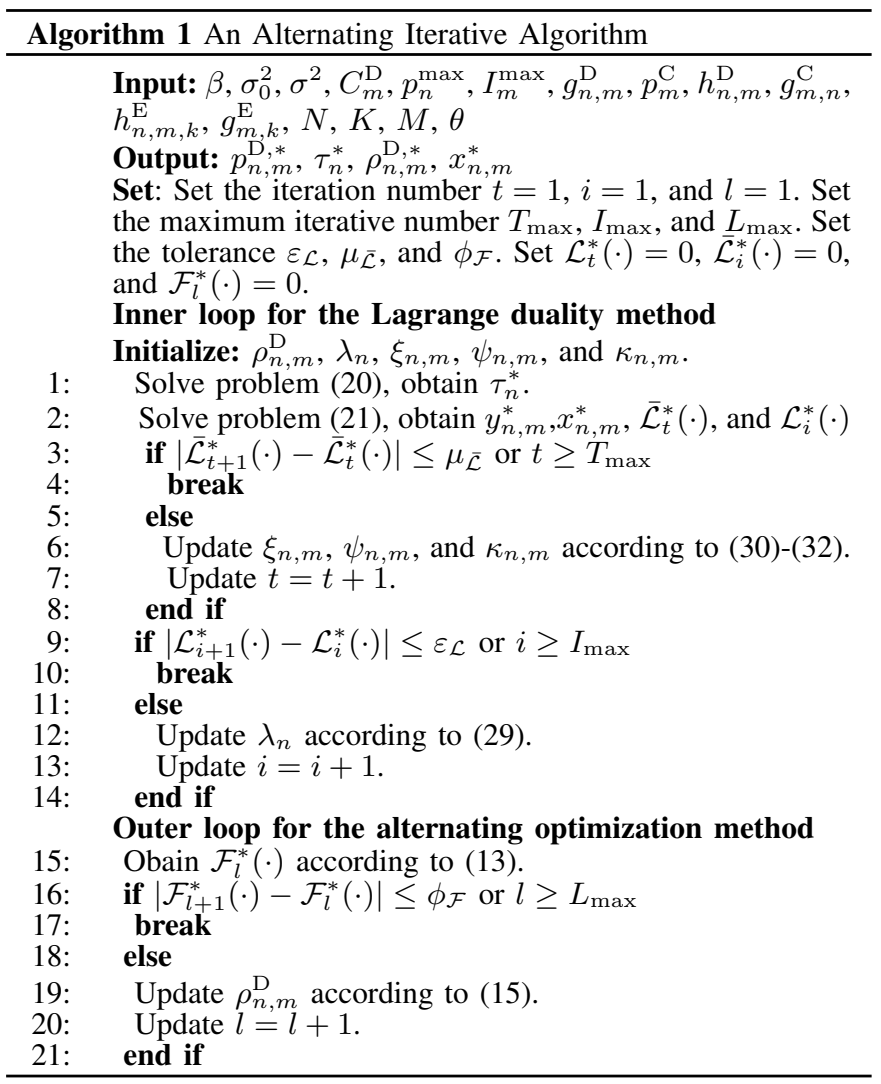

\section{Complexity Analysis}

The proposed algorithm is summarized in Algorithm 1. As can be seen, the proposed algorithm includes the inner loop and the outer loop. Defining $L_{\max }$ as the maximum iterative number of the outer loop, the complexity is $\mathcal{O}\left(L_{\max }\right)$. For the inner loop, it costs $\mathcal{O}(N M)$ for updating $\xi_{n, m}, \psi_{n, m}$, and $\kappa_{n, m}$, and $\mathcal{O}(N)$ for updating $\lambda_{n}$. Besides, defining $T_{\max }$ and $I_{\text {max }}$ as the maximum iterative number of $\mathcal{L}(\cdot)$ and $\overline{\mathcal{L}}(\cdot)$, we have $\mathcal{O}\left(M N^{2} I_{\max } T_{\max }\right)$. As a result, the overall complexity of the proposed algorithm is $\mathcal{O}\left(M N^{2} I_{\max } T_{\max } L_{\max }\right)$.

\section{NumERICAL RESUlTS}

In this section, simulation results are provided to show the effectiveness of the proposed algorithm. In the simulations, the coverage radius of the cellular network is $500 \mathrm{~m}$ and there are 3 CUs. There are 3 pairs of DUs and 3 EVEs. The channel gain is defined as $\chi d^{-3}$, where $\chi$ is the Rayleigh fading coefficient and $d$ is the inter-device distance. Other parameters include $\beta=0.6, C_{n}^{\mathrm{D}}=2 \mathrm{~mW}, \sigma_{0}^{2}=10^{-8} \mathrm{~mW}, \sigma^{2}=10^{-9} \mathrm{~mW}$, $p_{m}^{\mathrm{C}}=1 \mathrm{~W}, w=0.5, I_{m}^{\max }=0.5 \mathrm{~mW}, p_{n}^{\max }=20 \mathrm{~mW}$, $T_{\max }=I_{\max }=L_{\max }=10^{4}$, and $\varepsilon_{\mathcal{L}}=\mu_{\overline{\mathcal{L}}}=\phi_{\mathcal{F}}=10^{-5}$. To evaluate the fairness, Jain's fairness index (FI) is used [15], namely, FI $=\left(\sum_{n=1}^{N} R_{n}^{\mathrm{S}}\right)^{2} /\left(N \sum_{n=1}^{N}\left(R_{n}^{\mathrm{S}}\right)^{2}\right)$. Besides, the total SEE is defined as $\mathrm{SEE}=\sum_{n=1}^{N} R_{n}^{\mathrm{S}} / \sum_{n=1}^{N} E_{n}^{\mathrm{C}}$.

Fig. 2 shows the total SEE of DUs and Jain's FI versus the fairness factor $\alpha$ under different $p_{n}^{\max }$. It can be observed that both the total SEE of DUs and Jain's FI gradually increase when $p_{n}^{\max }$ becomes larger. The reason is that a larger $p_{n}^{\max }$ means that users can have more freedom to handle radio resources and the requests of different users can be satisfied more readily. Moreover, as $\alpha$ increases, Jain's FI monotonically increases, while the total SEE of DUs gradually decreases. That is to say, the larger $\alpha$ is, the more fair the resource allocation strategy obtained is, and the worse the system performance is. Besides, when $\alpha$ decreases, this trend is the opposite. This indicates that the desired fairness and system performance can be flexibly adjusted by choosing an appropriate $\alpha$.

For comparison, two benchmark algorithms are used, namely, zero fairness algorithm [6] and max-min fairness algorithm [19], [20]. Fig. 3 and Fig. 4 show the total SEE and Jain's FI versus $p_{n}^{\max }$ and $I_{m}^{\max }$ under different algorithms, respectively. With the increase of $p_{n}^{\max }$ and $I_{m}^{\max }$, both the total SEE of DUs and Jain's FI gradually increase and keep unchanged. The reason is that, when $p_{n}^{\max }$ is greater than $p_{n, m}^{\mathrm{D}, *}$, the steady-state environment of the system is no longer affected by the variation of $p_{n}^{\max }$. The same reason can also be usable for $I_{m}^{\max }$. Besides, zero fairness algorithm has the highest SEE of DUs, but it sacrifices the fairness. Contrastingly, max-min fairness algorithm, while providing excellent fairness, also dramatically degrades the system performance. Combining with Fig. 2, we can observe that the proposed algorithm can adaptively make a balance between the system performance and fairness, which is in contrast to benchmark algorithms. 


\section{CONCLusions}

In this paper, we investigated a resource allocation algorithm with $\alpha$ fairness in secure SWIPT-enabled D2D communication networks. A multi-objective problem was formulated to achieve the tradeoff between the secrecy rate and power consumption. The non-convex problem was transformed into a convex one, and closed-form solutions were derived. Simulation results demonstrated that the proposed algorithm had better performance than the conventional algorithms in terms of SEE and fairness.

\section{REFERENCES}

[1] Y. Xu, G. Gui, H. Gacanin, et al., "A survey on resource allocation for 5G heterogeneous networks: Current research, future trends and challenges," IEEE Commun. Surveys Tuts., vol. 23, no. 2, pp. 668-695, 2nd Quart. 2021.

[2] J. Huang, et al.,"Power allocation for D2D communications with SWIPT," IEEE Trans. Wireless Commun., vol. 19, no. 4, pp. 2308-2320, Apr. 2020.

[3] I. Budhiraja, N. Kumar, S. Tyagi, S. Tanwar and M. Guizani, "SWIPTenabled D2D communication underlaying NOMA-based cellular networks in imperfect CSI," IEEE Trans. Veh. Technol., vol. 70, no. 1, pp. 692-699, Jan. 2021.

[4] H. Yang, Y. Ye, X. Chu, et al., "Resource and power allocation in SWIPTenabled device-to-device communications based on a non-linear energy harvesting model," IEEE Internet Things J., vol. 7, no. 11, pp. 1081310825, Nov. 2020

[5] M. S. Khan, et al., "EPS-TRA: energy efficient peer selection and time switching ratio allocation for SWIPT-enabled D2D communication," IEEE Trans. Sustain. Comput., vol. 5, no. 3, pp. 428-437, Sept. 2020.

[6] J. Huang, J. Cui, C. Xing and H. Gharavi, "Energy-efficient SWIPTempowered D2D mode selection," IEEE Trans. Veh. Technol., vol. 69, no. 4, pp. 3903-3915, Apr. 2020.

[7] Y. Wang and L. Wang, "Matching theory-based cooperative secure transmission strategy for social-aware D2D communications," IEEE Trans. Veh. Technol., vol. 68, no. 10, pp. 10289-10294, Oct. 2019.

[8] H. Wang, B. Zhao and T. Zheng, "Adaptive full-duplex jamming receiver for secure D2D links in random networks," IEEE Trans. Commun., vol. 67, no. 2, pp. 1254-1267, Feb. 2019.

[9] X. Peng, H. Zhou, B. Qian, K. Yu, F. Lyu and W. Xu, "Enabling security-aware D2D spectrum resource sharing for connected autonomous vehicles," IEEE Internet of Things J., vol. 7, no. 5, pp. 3799-3811, May 2020.

[10] S. -H. Park and X. Jin, "Joint secure design of downlink and D2D cooperation strategies for multi-user systems," IEEE Signal Process. Lett., vol. 28, pp. 917-921, Apr. 2021.

[11] J. Ji, K. Zhu, D. Niyato and R. Wang, "Joint trajectory design and resource allocation for secure transmission in cache-enabled UAV-relaying networks with D2D communications," IEEE Internet of Things J., vol. 8, no. 3, pp. 1557-1571, Feb. 2021.

[12] Z. Song, Q. Ni, K. Navaie, S. Hou and S. Wu, "Energy- and spectralefficiency tradeoff with $\alpha$-fairness in downlink OFDMA systems," IEEE Commun. Lett., vol. 19, no. 7, pp. 1265-1268, Jul. 2015.

[13] Z. Kuang, L. Zhang and L. Zhao, "Energy- and spectral-efficiency tradeoff with $\alpha$-fairness in energy harvesting D2D communication," IEEE Trans. Veh. Technol., vol. 69, no. 9, pp. 9972-9983, Sept. 2020.

[14] D. Xu and Q. Li, "Resource allocation for secure communications in cooperative cognitive wireless powered communication networks," IEEE Systems J., vol. 13, no. 3, pp. 2431-2442, Sept. 2019.

[15] Z. Yang, W. Xu, Y. Pan, et al., "Optimal fairness-aware time and power allocation in wireless powered communication networks," IEEE Trans. Commun., vol. 66, no. 7, pp. 3122-3135, Jul. 2018.

[16] R. Li, Z. Wei, L. Yang, et al., "Resource allocation for secure multi-UAV communication systems with multi-eavesdropper," IEEE Trans. Commun., vol. 68 , no. 7, pp. 4490-4506, Jul. 2020.

[17] R. T. Marler and J. S. Arora, "The weighted sum method for multiobjective optimization: New insights," Struct. Multidiscipl. Optim., vol. 41, pp. 853-862, Jun. 2010.

[18] S. Boyd and L. Vandenberghe, Convex Optimization. Cambridge, U.K.: Cambridge Univ. Press, 2004.

[19] Y. Ye, L. Shi, X. Chu and G. Lu, "Throughput fairness guarantee in wireless powered backscatter communications with HTT," IEEE Wireless Commun. Lett., vol. 10, no. 3, pp. 449-453, Mar. 2021.

[20] D. Li, "Fairness-aware multiuser scheduling for finite-resolution intelligent reflecting surface-assisted communication," IEEE Commun. Lett., vol. 25, no. 7, pp. 2395-2397, Jul. 2021. 